\title{
BTLA mediates inhibition of human tumor-specific CD8+ T cells that can be partially reversed by vaccination
}

\author{
Laurent Derré, ${ }^{1}$ Jean-Paul Rivals, ${ }^{2}$ Camilla Jandus, ${ }^{1}$ Sonia Pastor, ${ }^{3}$ Donata Rimoldi, \\ Pedro Romero, ${ }^{1}$ Olivier Michielin, ${ }^{1,4}$ Daniel Olive, ${ }^{3}$ and Daniel E. Speiser ${ }^{1}$ \\ ${ }^{1}$ Ludwig Institute for Cancer Research, Lausanne, Switzerland. 2 University Hospital Center and University of Lausanne (CHUV), \\ Lausanne, Switzerland. 3INSERM UMR 891, Institut Paoli Calmettes, Marseille, France. \\ ${ }^{4}$ Multidisciplinary Oncology Center, Lausanne University Hospital (CHUV), Lausanne, Switzerland.
}

\begin{abstract}
The function of antigen-specific $\mathrm{CD8}^{+} \mathrm{T}$ cells, which may protect against both infectious and malignant diseases, can be impaired by ligation of their inhibitory receptors, which include CTL-associated protein 4 (CTLA-4) and programmed cell death 1 (PD-1). Recently, B and T lymphocyte attenuator (BTLA) was identified as a novel inhibitory receptor with structural and functional similarities to CTLA-4 and PD-1. BTLA triggering leads to decreased antimicrobial and autoimmune $T$ cell responses in mice, but its functions in humans are largely unknown. Here we have demonstrated that as human viral antigen-specific $\mathrm{CD8}^{+} \mathrm{T}$ cells differentiated from naive to effector cells, their surface expression of BTLA was gradually downregulated. In marked contrast, human melanoma tumor antigen-specific effector $\mathrm{CD8}^{+} \mathrm{T}$ cells persistently expressed high levels of BTLA in vivo and remained susceptible to functional inhibition by its ligand herpes virus entry mediator (HVEM). Such persistence of BTLA expression was also found in tumor antigen-specific $\mathrm{CD8}^{+} \mathrm{T}$ cells from melanoma patients with spontaneous antitumor immune responses and after conventional peptide vaccination. Remarkably, addition of CpG oligodeoxynucleotides to the vaccine formulation led to progressive downregulation of BTLA in vivo and consequent resistance to BTLA-HVEM-mediated inhibition. Thus, BTLA activation inhibits the function of human CD8 ${ }^{+}$ cancer-specific $T$ cells, and appropriate immunotherapy may partially overcome this inhibition.
\end{abstract}

\section{Introduction}

Activation of lymphocytes is controlled by 2 classes of signals: first, by those triggered through the $\mathrm{T}$ cell receptor upon interaction with antigenic peptide bound to MHC molecules; and second, by signals delivered by binding of coreceptors to their ligands on antigen-presenting cells (1). Coreceptors consist of costimulatory and coinhibitory receptors (2-7). Preclinical and clinical data indicate that the co-inhibitory receptors CTL-associated protein 4 (CTLA-4) and programmed cell death 1 (PD-1) are co-responsible for the suppression of human effector $\mathrm{T}$ cell responses to infectious diseases and cancer $(5,6)$; the therapeutic blockade of these 2 pathways is in promising clinical development. Lymphocytes can express additional inhibitory receptors, such as killer-inhibitory receptors and C-lectin-type receptors (8) - both of these classes, however, are expressed by only small subsets of $\mathrm{T}$ cells $(8,9)$.

A more recently described co-inhibitory receptor is $\mathrm{B}$ and $\mathrm{T}$ lymphocyte attenuator (BTLA; CD272), an immunoglobulin-like molecule belonging to the CD28:B7 family, which is expressed by the majority of lymphocytes $(6,10-12)$. Interestingly, its ligand, herpes virus entry mediator (HVEM), is a member of the TNF receptor (TNFR) superfamily $(10,11)$. This receptor system also includes lymphotoxin- $\alpha$, LIGHT (CD258), and CD160, which are present at the cytoplasmic membrane of cells of different histological origin. They may compete for their ligand HVEM, which is present on T, B, and NK cells, DCs, and myeloid cells, and also a variety of tumor cells (6, 10-12). The ligation of BTLA by HVEM leads to phosphorylation of immunoreceptor tyrosine-based inhibition motifs (ITIMs) and

Conflict of interest: The authors have declared that no conflict of interest exists. Citation for this article: J. Clin. Invest. 120:157-167 (2010). doi:10.1172/JCI40070.
Src homology 2 (SH2) domain-containing phosphatase 1 (SHP-1)/ SHP-2 association, resulting in decreased $\mathrm{T}$ cell proliferation and cytokine production (12-14). In B cells, BTLA regulates B cell receptor signaling by reducing the phosphorylation of syk, B cell linker protein (BLNK), and phospholipase C $\gamma 2$ (PLC $\gamma 2)(15)$.

$\mathrm{B}$ and $\mathrm{T}$ cell development is normal in BTLA-deficient mice. Mature lymphocytes, however, are functionally altered and show enhanced generation of memory $\mathrm{T}$ cells and memory responses (16). BTLA deficiency was found to enhance protection from murine malaria (17) and to aggravate experimental autoimmune encephalomyelitis (12) and allergic airway inflammation (18) and was associated with spontaneous development of an autoimmune hepatitis-like disease (19). More recently, BTLA has been shown to be involved in peripheral $\mathrm{T}$ cell tolerance induction (20) and in early control of tissue damage and of antibacterial immunity (21).

In humans, BTLA expression may be altered by specific immunotherapy with allergens, as shown for allergic rhinitis (22). However, only little is known about the role and function of BTLA in humans, and there are no data yet available on antigen-specific $\mathrm{T}$ cells. In this study, we show for the first time to our knowledge that BTLA is downregulated during human $\mathrm{CD}^{+} \mathrm{T}$ cell differentiation to effector cells. This was, however, not the case for tumor antigen-specific (Melan-AMART-1-specific) T cells, which persistently expressed BTLA despite effector cell differentiation in unvaccinated melanoma patients. In contrast, when CpGs were used as adjuvant for vaccination, Melan-A ${ }^{\mathrm{MART}-1}$-specific T cells downregulated BTLA, developed strong effector functions, and became independent of BTLA-mediated inhibition. Finally, the BTLA ligand HVEM was found to be expressed by melanoma tumors in situ and mediated functional inhibition of $\mathrm{BTLA}^{+} \mathrm{T}$ cells. 


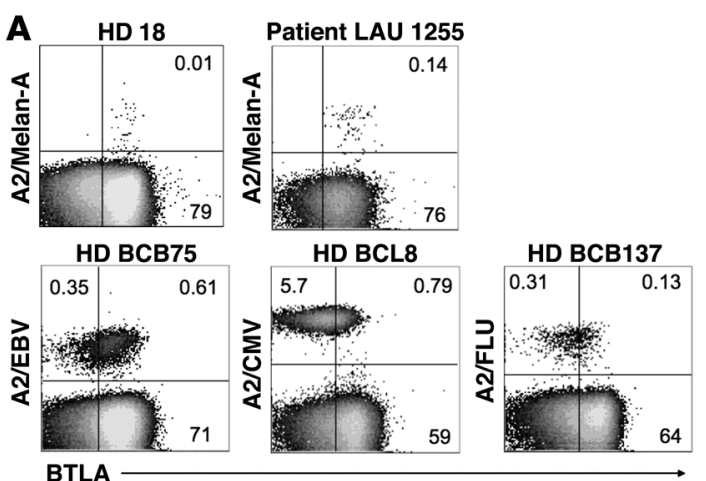

C Total $\operatorname{CD8}(\mathrm{GMFI}=25)$

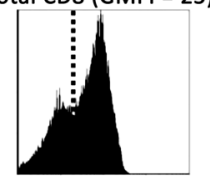

$\mathrm{CM}(\mathrm{GMFI}=91)$

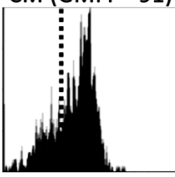

$\mathrm{N}(\mathrm{GMFI}=106)$

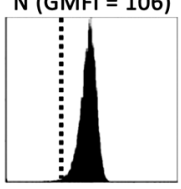

$\mathrm{EM}(\mathrm{GMFI}=31)$

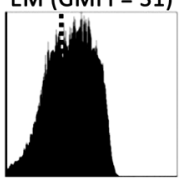

EMRA $(\mathrm{GMFI}=6$
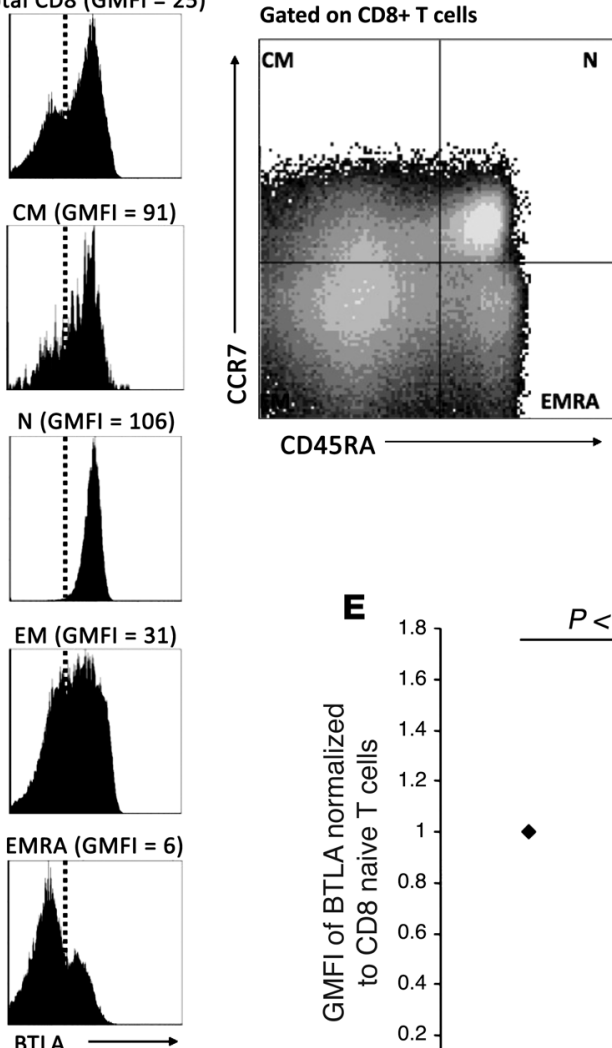

E
B

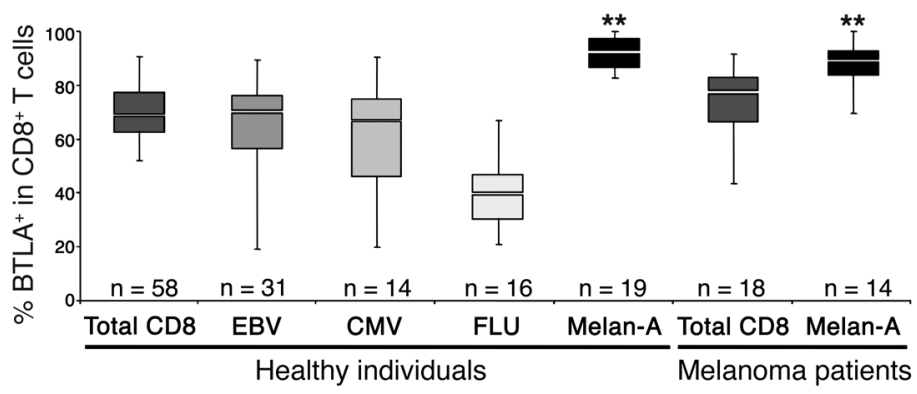

D

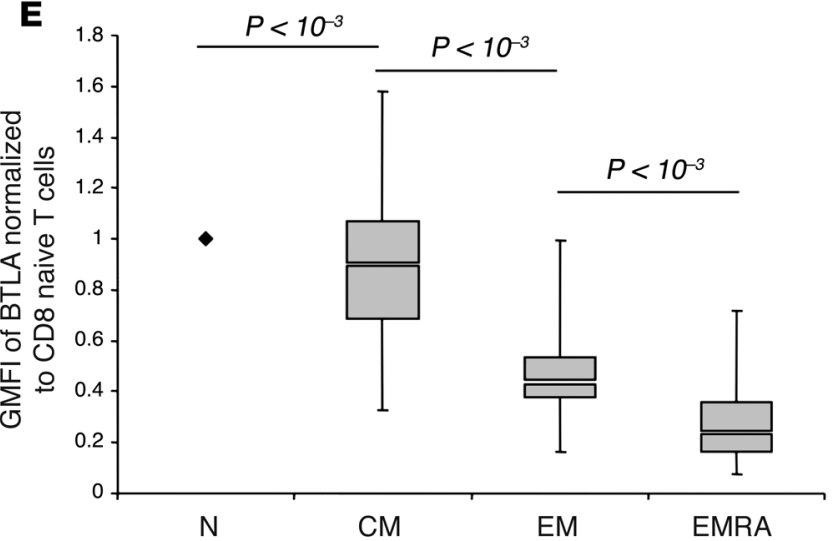

Figure 1

BTLA (CD272) is downregulated with progressive CD8 ${ }^{+} \mathrm{T}$ cell differentiation, except in tumor antigen-specific CD8 $8^{+} \mathrm{T}$ cells. Direct ex vivo analysis of CD8 ${ }^{+} T$ cells from PBMCs by flow cytometry. (A) Representative examples of BTLA expression by Melan-AMART-1-and virus-specific (EBV, CMV, and influenza virus [FLU]) $\mathrm{CD}^{+} \mathrm{T}$ cells from healthy donors (HD) and melanoma patients. BTLA+ $T$ cells were distinguished from BTLA $^{-}$T cells using a threshold established according to the autologous naive T cell subset, which is always BTLA positive. Numbers indicate percentage of positive or negative cells. (B) Comparison of BTLA expression on total CD8 ${ }^{+}$and virus- and Melan-A-specific T cells, from healthy individuals and unvaccinated melanoma patients. ${ }^{*} P<10^{-2}$ for each of the 2 populations of Melan-A-specific T cells compared with all 5 other populations. (C-E) BTLA expression in subsets of $C D 8^{+} \mathrm{T}$ cells of healthy individuals $(n=58)$, upon definition of early and late differentiation stages by CD45RA and CCR7 expression (i.e. naive [N, CD45RA+CCR7 ${ }^{+}$], central memory [CM, CD45RA-CCR7 ${ }^{+}$], effector memory [EM, CD45RA-CCR7-], and effector memory RA ${ }^{+}$[EMRA, CD45RA ${ }^{+}$CCR7-] cells), gated as shown in C; a representative example demonstrates the narrow positive peak observed in phenotypic naive cells. (D) Statistical assessment of BTLA+ CD8 ${ }^{+}$T cell subsets. (E) GMFI data, showing results compatible with those presented in D. Overall GMFI was determined by including the whole range from BTLA-negative to -positive cells, as shown in C. GMFI data were normalized to values determined in autologous naive CD8+ $\mathrm{T}$ cells. 


\section{Results}

Virus- but not tumor-specific effector $C D 8^{+}$T cells downregulate BTLA. To investigate the expression of BTLA by tumor-specific (Melan-AMART-1) and virus-specific (EBV, CMV, and influenza virus) human CD8 ${ }^{+}$ $\mathrm{T}$ cells, we performed ex vivo multicolor flow cytometry analysis on PBMCs from healthy donors and untreated melanoma patients (Figure 1A). In healthy individuals, Melan-A ${ }^{\text {MART-1}}{ }_{\text {-specific }} \mathrm{CD}^{+}$ $\mathrm{T}$ cells were predominantly BTLA ${ }^{+}$(Figure $1 \mathrm{~B}$ ), with significantly higher BTLA expression than on virus-specific T cells or on total $\mathrm{CD}^{+} \mathrm{T}$ cells. This could be explained by their differentiation stages, since Melan-A $\mathrm{A}^{\mathrm{MART}-1}$-specific $\mathrm{T}$ cells are almost always naive in healthy donors (Supplemental Figure 1; supplemental material available online with this article; doi:10.1172/JCI40070DS1), in contrast to virus-specific CD8 $8^{+} \mathrm{T}$ cells (23). Correspondingly, naive $T$ cells are BTLA positive in the murine system $(11,24,25)$. Therefore, we studied BTLA expression on total $\mathrm{CD}^{+} \mathrm{T}$ cells, in relation to their differentiation stage as defined by expression of CCR7 and CD45RA (26) in 58 healthy individuals (Figure 1, C and $\mathrm{D})$. Almost all phenotypic naive $\mathrm{T}$ cells $(\mathrm{N})$ and the majority of central-memory $\mathrm{T}$ cells (CM) expressed BTLA. In contrast, effector memory $\mathrm{T}$ cells (EM) and effector memory RA ${ }^{+} \mathrm{T}$ cells (EMRA) were frequently BTLA negative, demonstrating decreased BTLA expression correlating with $\mathrm{CD}^{+} \mathrm{T}$ cell differentiation.

In many instances, BTLA receptor levels displayed a pattern of continuous surface expression without a clear transition from negative to positive T cells. However, phenotypically naive CD8 ${ }^{+}$ $\mathrm{T}$ cells were consistently positive, with a distinct narrow peak. We therefore used this population as a reference to set the threshold for $\mathrm{BTLA}^{+}$cells (Figure 1C). Moreover, we also evaluated the data in terms of geometric mean fluorescence intensity (GMFI) values normalized to the GMFI of the autologous naive $\mathrm{CD}^{+} \mathrm{T}$ cell subset. The results (Figure 1E) closely resembled those based on the assessment of percentage of $\mathrm{BTLA}^{+}$cells (Figure 1D). Thus, using autologous naive $\mathrm{CD}^{+} \mathrm{T}$ cells as the internal BTLA reference allowed consistent standardization and normalization of both percentage and GMFI data.

Analyzing PBMCs of melanoma patients revealed that BTLA expression was high among Melan-A $\mathrm{A}^{\mathrm{MART}-1}$-specific $\mathrm{T}$ cells, surprisingly even among memory and effector cells (Figure 1B and Figure 2, A and B). In contrast, total $\mathrm{CD}^{+} \mathrm{T}$ cells showed reduced BTLA expression, with progressive differentiation to effector cells (Figure 2B).

For comprehensive immune characterization, it is also important to investigate $\mathrm{T}$ cells from diseased organs/tissues. Therefore, we analyzed lymphocytes from metastatic lymph nodes directly ex vivo and assessed BTLA expression by Melan- $A^{\text {MART-1 }}{ }_{\text {-specific } T}$ cells as found in 13 of 18 normal lymph nodes and in 6 of 6 tumorinfiltrated lymph nodes. Once again, the Melan- $\mathrm{A}^{\mathrm{MART}-1}-\mathrm{specific}$ memory and effector $\mathrm{T}$ cells were largely $\mathrm{BTLA}^{+}$, and the remaining $\mathrm{CD}^{+} \mathrm{T}$ cells bore significantly lower levels of BTLA (Figure 2, $\mathrm{C}$ and D). Thus, the expression of the inhibitory receptor BTLA was high for Melan-A $\mathrm{A}^{\mathrm{MART}-1}$-specific T cells both in circulation and in solid tissue, despite the fact these cells engage in considerable "spontaneous," disease-driven responses in most patients with progressive melanoma (27).

In situ expression of HVEM by melanoma cells and $T$ cell inhibition by BTLA-HVEM ligation. We next sought to determine whether BTLA could negatively regulate $T$ cell function via binding to HVEM expressed by cancer cells. The characterization of cultured melanoma cell lines (Figure 3A) revealed that 26 of 40 expressed
HVEM (19 of 40 highly and 7 of 40 weakly), whereas none of them expressed BTLA or LIGHT (Figure 3B). Subsequently, we assessed in situ HVEM expression by immunohistochemistry on paraffinembedded metastases from patients (Figure 3C). We found that $50 \%$ and $25 \%$ of metastases were strongly and moderately positive for HVEM expression, respectively, while the remaining $25 \%$ were weakly positive or negative (Table 1 ).

To test whether HVEM expression by melanoma cells could inhibit the function of antigen-specific T cells, we analyzed IFN- $\gamma$ production by Melan-A ${ }^{\text {MART-1}}{ }_{-}$specific $\mathrm{CD}^{+} \mathrm{T}$ cell clones upon triggering with HVEM-expressing melanoma cell lines. For these experiments, we first characterized our new mAb, BTLA-8.2, and found that it efficiently blocked the interaction between BTLA and HVEM (Supplemental Figure 2A). Subsequent experiments showed that $\mathrm{T}$ cell clones properly secreted IFN- $\gamma$ in the presence of IgG1 control antibody, as expected. In contrast, IFN- $\gamma$ production was significantly increased for clones expressing BTLA upon addition of blocking mAb BTLA-8.2 during the stimulation. In contrast, no change in IFN- $\gamma$ secretion by a clone lacking BTLA expression was observed (clone 618-420, Figure 3D). These data demonstrate functional inhibition of tumor-specific $\mathrm{CD}^{+} \mathrm{T}$ cells via BTLA-HVEM interactions.

Downregulation of BTLA upon vaccination with peptide and Cp G. Next, we addressed whether vaccination of melanoma patients altered BTLA expression by tumor antigen-specific $\mathrm{CD}^{+} \mathrm{T}$ cells. Patients received s.c. vaccinations with Melan- $\mathrm{A}^{\mathrm{MART}-1}$ peptides in conventional vaccine formulations, e.g., emulsified in incomplete Freund's adjuvant (IFA) (28). A second cohort of patients was treated similarly, except that CPG oligonucleotides (PF-3512676) were added to the vaccine formulation, resulting in considerably enhanced $\mathrm{CD}^{+} \mathrm{T}$ cell responses, as reported previously (29). Representative multicolor flow cytometry analyses of PBMCs are depicted in Figure 4A. Interestingly, while BTLA expression by Melan-A ${ }^{\text {MART-1 }}$ specific $T$ cells persisted at high levels after vaccination without CPG, BTLA downregulation was observed after vaccination with CPG (Figure 4, A and B), which occurred in the EM/EMRA subsets (Figure 4C). For total CD8 ${ }^{+} \mathrm{T}$ cells, no significant differences in BTLA expression were observed between healthy donors and (un)treated patients (Supplemental Figure 3). These data demonstrate that vaccinations with CPG lead to BTLA downregulation on tumor antigen-specific $T$ cells.

BTLA downregulation correlates with the number of monthly booster vaccinations with $C p G$. After vaccination with $C p G$, BTLA downregulation on Melan-A ${ }^{\mathrm{MART}-1}$-specific CD8 T lymphocytes differed considerably among patients (Figure 4B). Interestingly, we found a correlation with the number of monthly booster vaccinations. Percentages of BTLA expression by Melan- $\mathrm{A}^{\mathrm{MART}-1}$-specific T cells were lowest in patients after multiple vaccinations, whereas low numbers (2-4) of vaccinations were not sufficient to induce significant BTLA downregulation (Figure 5, A and B). The reduction in BTLA expression was again a feature of EM/EMRA cells (Figure 5C). In addition, we found a tight correlation between BTLA and CD28 downregulation $(P<0.001$, Supplemental Figure 4A), the latter of which is characteristic of advanced effector $T$ cell differentiation (2). No significant correlation was found between the number of vaccinations and BTLA expression by total $\mathrm{CD}^{+} \mathrm{T}$ cells and their EM/ EMRA subsets (Supplemental Figure 4B), suggesting that there is no major bystander effect of $\mathrm{CPG}$ vaccinations on BTLA expression. These findings likely reflect the fact that the vaccinations resulted in strong activation of Melan- $\mathrm{A}^{\mathrm{MART}-1}{ }_{-}$-specific $\mathrm{T}$ cells but not $\mathrm{T}$ cells 
A
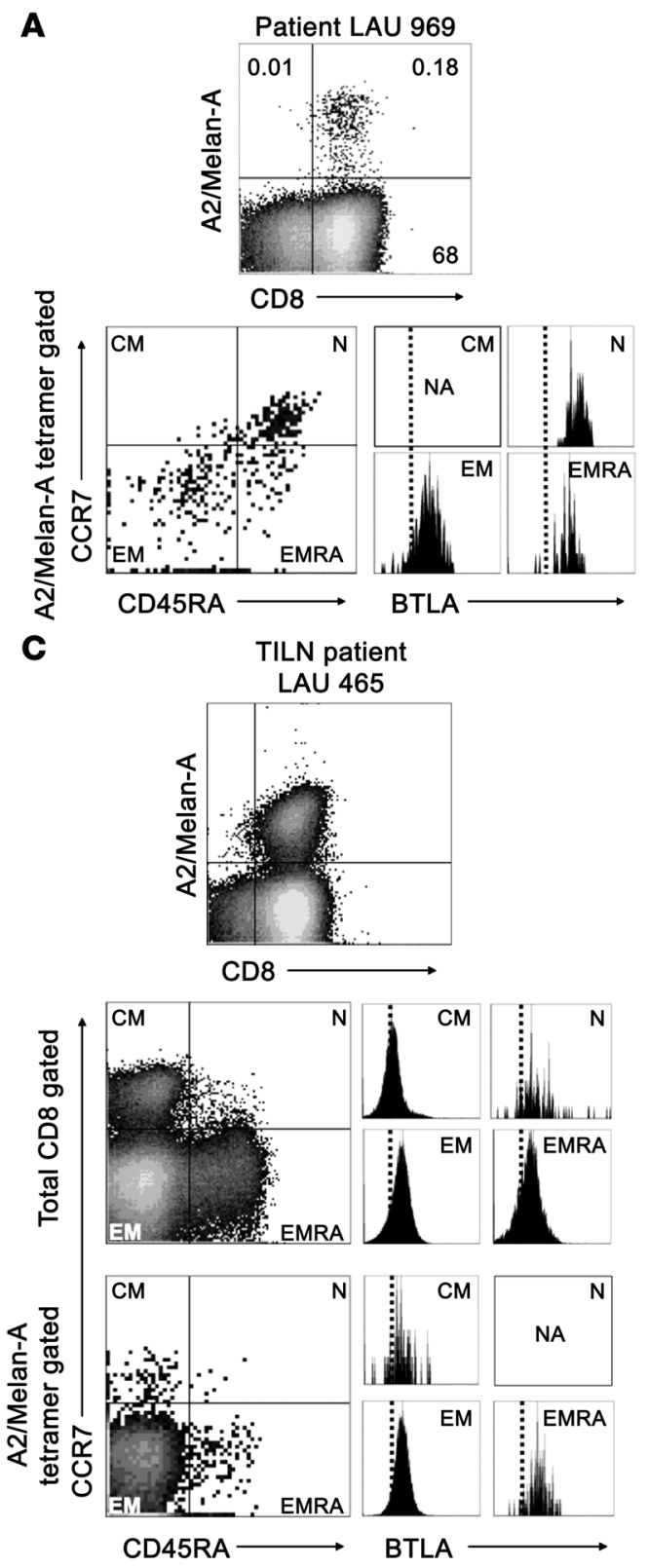

B

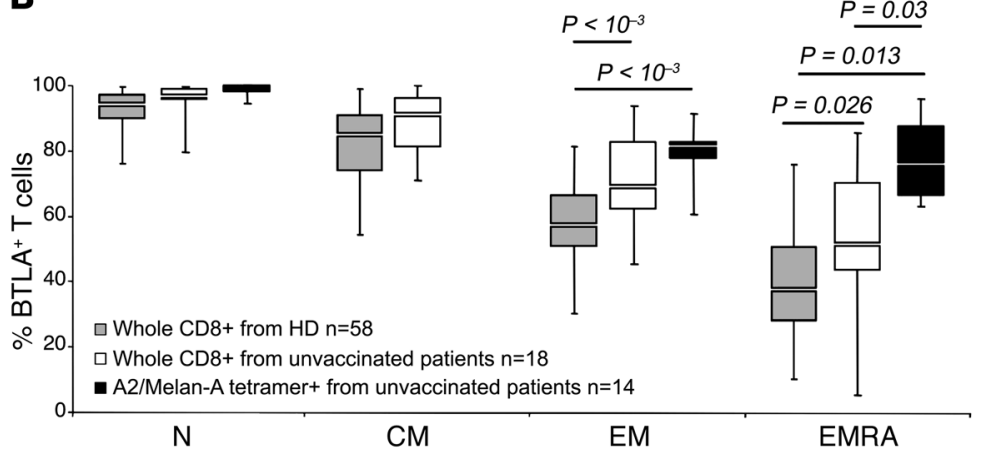

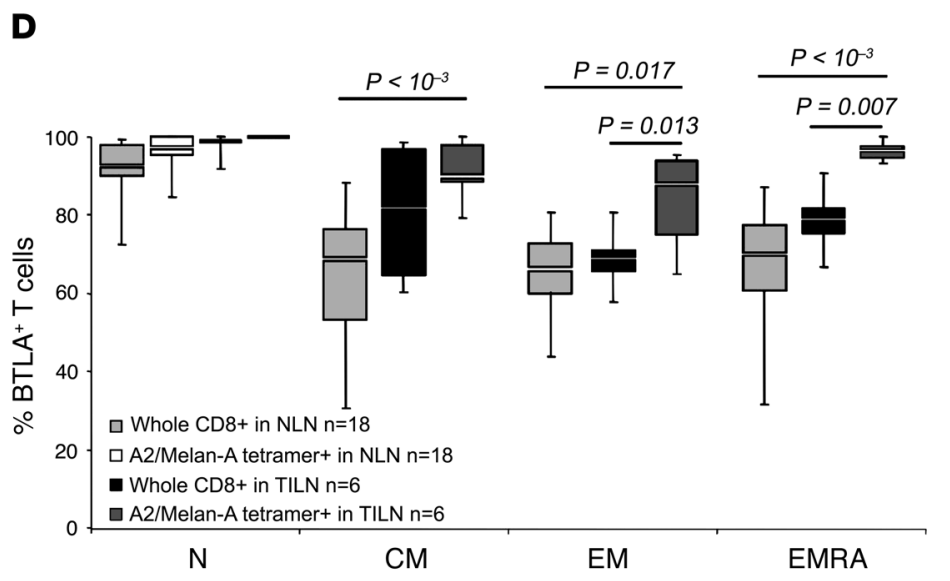

Figure 2

Persistence of BTLA expression on Melan-AMART-1_specific lymphocytes despite effector cell differentiation. (A) Analysis of BTLA expression by Melan-AMART-1-specific T cells depending on differentiation. Representative example of PBMCs from an unvaccinated melanoma patient. (B) Percentages of BTLA+ CD8 ${ }^{+}$T cells from healthy individuals (gray, $n=58$ ) and unvaccinated melanoma patients (white, $n=18$ ); and from Melan-AMART-1 tetramer ${ }^{+} \mathrm{CD}^{+}$T cells from unvaccinated patients (black, $n=14$ ). Data from healthy donors (same as in Figure 1D) are shown again for comparison. Circulating central memory Melan-AMART-1_specific cells were rare, precluding analysis for BTLA expression. (C and D) BTLA expression by CD45RA ${ }^{+-}$and CCR7 $7^{+-}$T cells from normal lymph nodes (NLN, $n=18$ ) and tumor-infiltrated lymph nodes (TILN, $\left.n=6\right)$ from melanoma patients. (C) Representative example and (D) comparison of BTLA expression in CD8 ${ }^{+}$T cells and Melan-AMART-1-specific T cells. In NLNs, Melan-AMART-1-specific T cells are all naive. Light gray, whole CD8 ${ }^{+}$T cells in NLNs $(n=18)$; white, Melan-AMART-1 tetramer ${ }^{+}$CD8 ${ }^{+}$T cells in NLNs $(n=18)$; black, whole CD8 ${ }^{+}$T cells in TILNs $(n=6)$; dark gray, Melan-AMART-1 tetramer ${ }^{+}$CD8 ${ }^{+}$T cells in TILNs $(n=6)$.

with other antigen specificities (data not shown). In conclusion, repeated vaccination with peptide and $\mathrm{CPG}$ leads to progressive BTLA downregulation by vaccine-specific $\mathrm{CD}^{+} \mathrm{T}$ cells.

Regulation of proliferation and cytokine production of primary $C D 8^{+}$ $T$ cells depending on BTLA expression and interaction with HVEM. We next determined whether BTLA expression by Melan-AMART-1_ specific $\mathrm{T}$ cells was associated with functional inhibition of $\mathrm{T}$ cells, as assessed ex vivo and in vitro. First, we examined the relationship between the in vivo frequency of Melan- $A^{\text {MART- } 1}-$ specific $\mathrm{CD}^{+} \mathrm{T}$ cells and their proportion of $\mathrm{BTLA}^{+}$cells in PBMCs from patients vaccinated without or with $\mathrm{CPG}$. High $\mathrm{T}$ cell frequencies as well as low percentages of $\mathrm{BTLA}^{+}$cells were rarely 
A
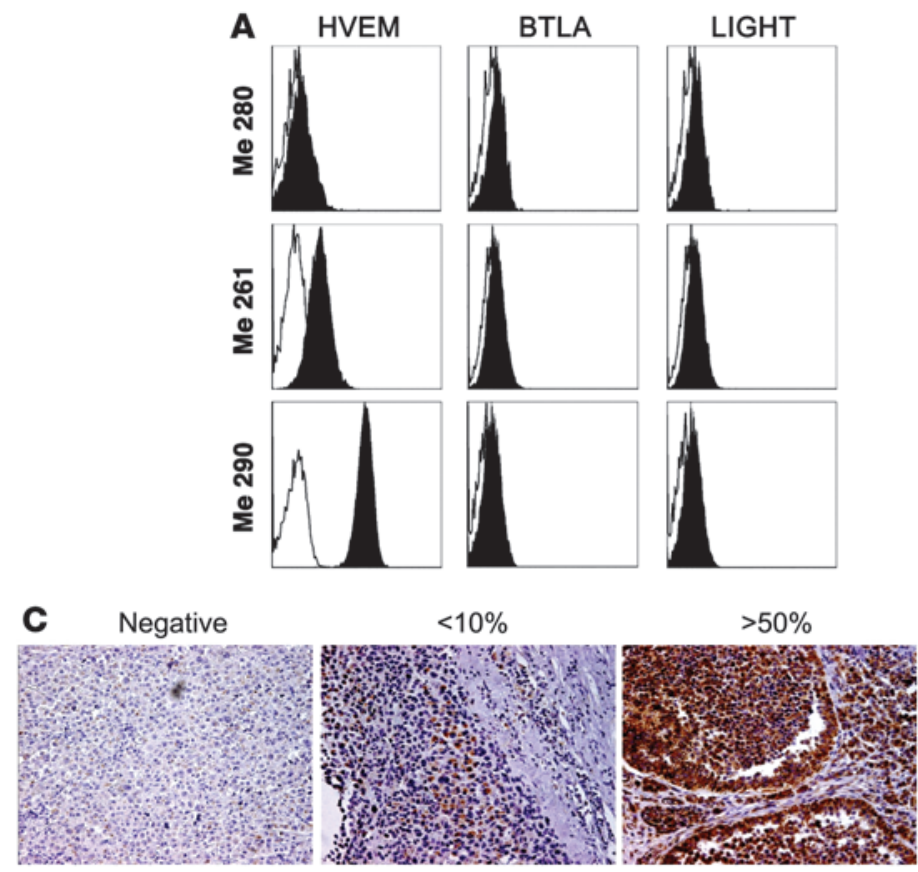

B

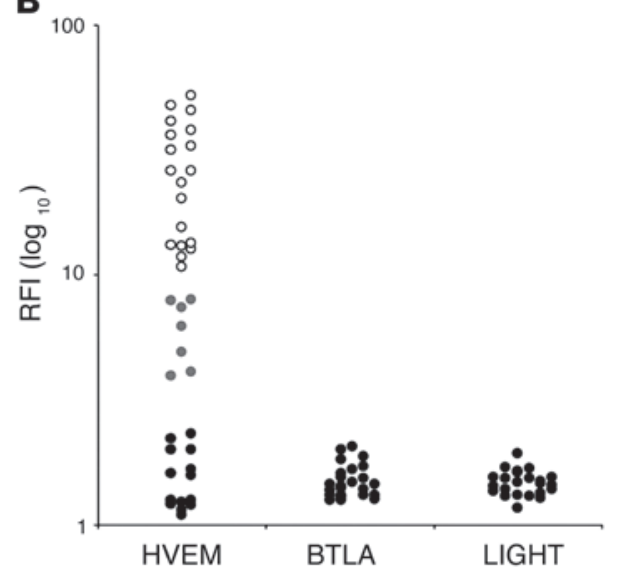

D

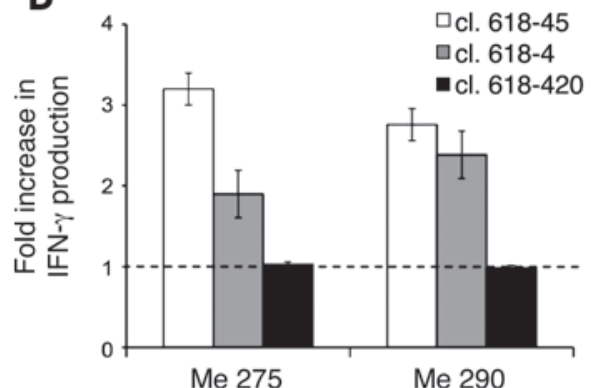

cl. $618-45$

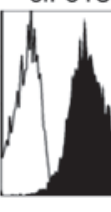

cl. $618-4$

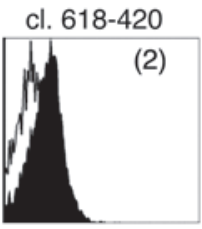

BTLA

Figure 3

Expression of HVEM and its ligands BTLA and LIGHT by melanoma cells; and BTLA-mediated functional inhibition of tumor antigen-specific $\mathrm{BTLA}^{+}$CD8 ${ }^{+}$T cells. (A) Representative examples of BTLA, LIGHT, and HVEM expression by the melanoma cell lines Me 280, Me 261, and Me 290. Cells were stained with isotype control (open histograms) or with anti-HVEM, anti-BTLA, or anti-LIGHT mAb (filled histograms) (B) Relative expression of HVEM, BTLA, and LIGHT by melanoma cell lines, expressed as ratio fluorescence intensity (RFI), i.e., MFI with specific $\mathrm{mAb} / \mathrm{MFI}$ with isotype control. Of 40 melanoma cell lines analyzed, 19 were highly positive (white dots), 7 weakly positive (gray dots), and 14 negative for HVEM expression (black dots). None of the melanoma cell lines expressed BTLA or LIGHT (black dots). (C) HVEM expression (in reddish brown) detected in paraffin-embedded tumor sections from 16 tumors of 14 melanoma patients. Examples show tumor tissues that were HVEM-negative, weakly positive $\left(<10 \% \mathrm{HVEM}^{+}\right.$tumor cells) or strongly positive $(>50 \%$ positive tumor cells). Original magnification, $\times 200$. (D) Melan-AMART-1/HLA-A ${ }^{*} 0201-$ specific CD8 ${ }^{+} \mathrm{T}$ cell clones (cl.) 618-45, 618-4, and 618-420 were stimulated by melanoma cell lines Me 275 and Me 290 (both Melan- $\mathrm{A}^{+}, \mathrm{HLA}-\mathrm{A}^{*} 0201^{+}, \mathrm{HVEM}^{+}$). The graph shows fold increase in IFN- $\gamma$ production by Melan- $\mathrm{A}^{\text {MART-1}}$-specific clones in the presence of blocking antibody BTLA-8.2, relative to isotype control antibody. IFN- $\gamma$ production was determined by ELISA in supernatants of 24-hour cultures. BTLA expression by T cell clones was assessed by flow cytometry with BTLA-specific antibody (filled histograms) and isotype control (open histograms). GMFI is indicated in parentheses.

observed without $\mathrm{CPG}$ but occurred frequently with CpG (Figure 6A). Consequently, the 2 parameters correlated significantly $(P=0.012)$ after vaccination with but not without $\mathrm{CpG}$. Second, we analyzed the in vitro proliferation of Melan-A $\mathrm{A}^{\mathrm{MART}-1}$-specific $\mathrm{CD}^{+} \mathrm{T}$ cells. After 10 -day stimulation by autologous mature DCs loaded with Melan-AMART-1 peptide, expansion of HLA-A2/ Melan-A MART- 1 tetramer ${ }^{+} \mathrm{CD}^{+} \mathrm{T}$ cells was significantly increased in the presence of blocking mAb BTLA-8.2 (Figure 6B), showing that BTLA inhibited proliferation of $\mathrm{CD}^{+} \mathrm{T}$ cells. Third, we measured ex vivo cytokine secretion by primary $\mathrm{CD} 8^{+} \mathrm{T}$ cells. PBMCs from healthy individuals and patients were triggered for 4 hours with SKMEL37 melanoma cells transfected by vector encoding HVEM, or empty vector (Supplemental Figure 5), which were loaded (or not) with cognate peptide. For both Melan-AMART-1 and EBV-specific T cells expressing high levels of BTLA, IFN- $\gamma$ as well as TNF- $\alpha$ secretion was reduced when melanoma cell lines expressed HVEM (Figure 6, C and D). This inhibition correlated significantly with percentages of $\mathrm{T}$ cells expressing BTLA (Figure 6E). In contrast to IFN- $\gamma$ and TNF- $\alpha$, CD107a (a marker for cytotoxicity; ref. 30) was not affected by HVEM expressed by SKMEL37 cells (Supplemental Figure 6). Consistent with this finding, cytotoxicity was not altered (data not shown). Together, 


\section{Table 1}

HVEM expression analyzed by immunohistochemistry

\begin{tabular}{lcccc} 
HVEM expression & Negative & $<\mathbf{1 0} \%$ & $\mathbf{1 0} \% \mathbf{- 5 0} \%$ & $>\mathbf{5 0} \%$ \\
Number of tumors $(n=16)$ & 1 & 3 & 4 & 8 \\
Percentage of tumors & 6.25 & 18.75 & 25 & 50 \\
\hline
\end{tabular}

The table summarizes all results from immunohistochemical HVEM staining of paraffin-embedded tumor sections of 16 tumors from 14 patients with melanoma.

these data reveal an inhibitory role for BTLA in $\mathrm{CD}^{+} \mathrm{T}$ cell proliferation and cytokine production but not cytotoxicity.

Modulation of BTLA expression by HVEM. We observed that TCR ligation by $\mathrm{HVEM}^{+}$stimulator cells led to downregulation of BTLA on antigen-specific T cells (Figure 7, A and B). Kinetic studies revealed that $\mathrm{HVEM}^{+}$stimulator cells induced a rapid BTLA downregulation between 4 hours and 2 days, independent of peptide antigen (Figure 7C). In contrast, in the absence of HVEM, antigen-triggered BTLA downregulation was less pronounced and led to higher BTLA expression levels at the late time points. Surface BTLA expression returned to basal levels in all conditions after 4-6 days (Figure 7C). These results indicate that BTLA is reversibly downregulated upon binding to its ligand HVEM. In parallel, we observed that the yield of antigen-specific $\mathrm{CD}^{+} \mathrm{T}$ cells was higher in the absence of HVEM (Figure 7D), confirming the BTLA-HVEM-mediated inhibition of $\mathrm{CD}^{+} \mathrm{T}$ cell proliferation.

\section{Discussion}

BTLA is widely expressed by human $\mathrm{CD}^{+} \mathrm{T}$ cells but progressively downregulated with advanced effector $\mathrm{T}$ cell differentiation, with the majority of long-term effector T cells being BTLAnegative (Figure 1, D and E). In a parallel study, we found high BTLA expression in CMV-specific T cells during the early phase of acute $\mathrm{CMV}$ infection and consequent $\mathrm{T}$ cell inhibition via BTLA (D. Olive, unpublished observation). In the present study, we focused on later phases of immune responses, i.e., in individuals with persistent or resolved viral infections or malignant diseases and patients after repeated vaccinations. While we observed BTLA downregulation in virus-specific T cells and tumor-specific T cells after CpG-based vaccination, persistence of BTLA expression was seen in melanoma patients that were untreated or after vaccination without CpG. Sizeable fractions of T cells remained BTLA ${ }^{+}$, supporting the development of therapeutic interventions interfering with BTLA-mediated immune regulation.

Recent advances in $\mathrm{T}$ cell vaccination have enabled the generation of larger numbers of tumor antigen-specific $T$ cells and broader immune responses, but with limited clinical responses. In persistent or progressive disease, immune protection may be hampered by several mechanisms of peripheral $\mathrm{T}$ cell inhibition $(3,31)$. Our data indicate that inhibition via BTLA may play a central role, since it is persistently expressed by tumor-specific $\mathrm{CD}^{+}$ T lymphocytes (Figures 1 and 2). BTLA expression is maintained at high levels despite differentiation to effector cells, unlike in virus-specific $\mathrm{CD}^{+} \mathrm{T}$ cells (Figure 1 and data not shown). However, significant proportions of EBV-and CMV-specific CD8 ${ }^{+} \mathrm{T}$
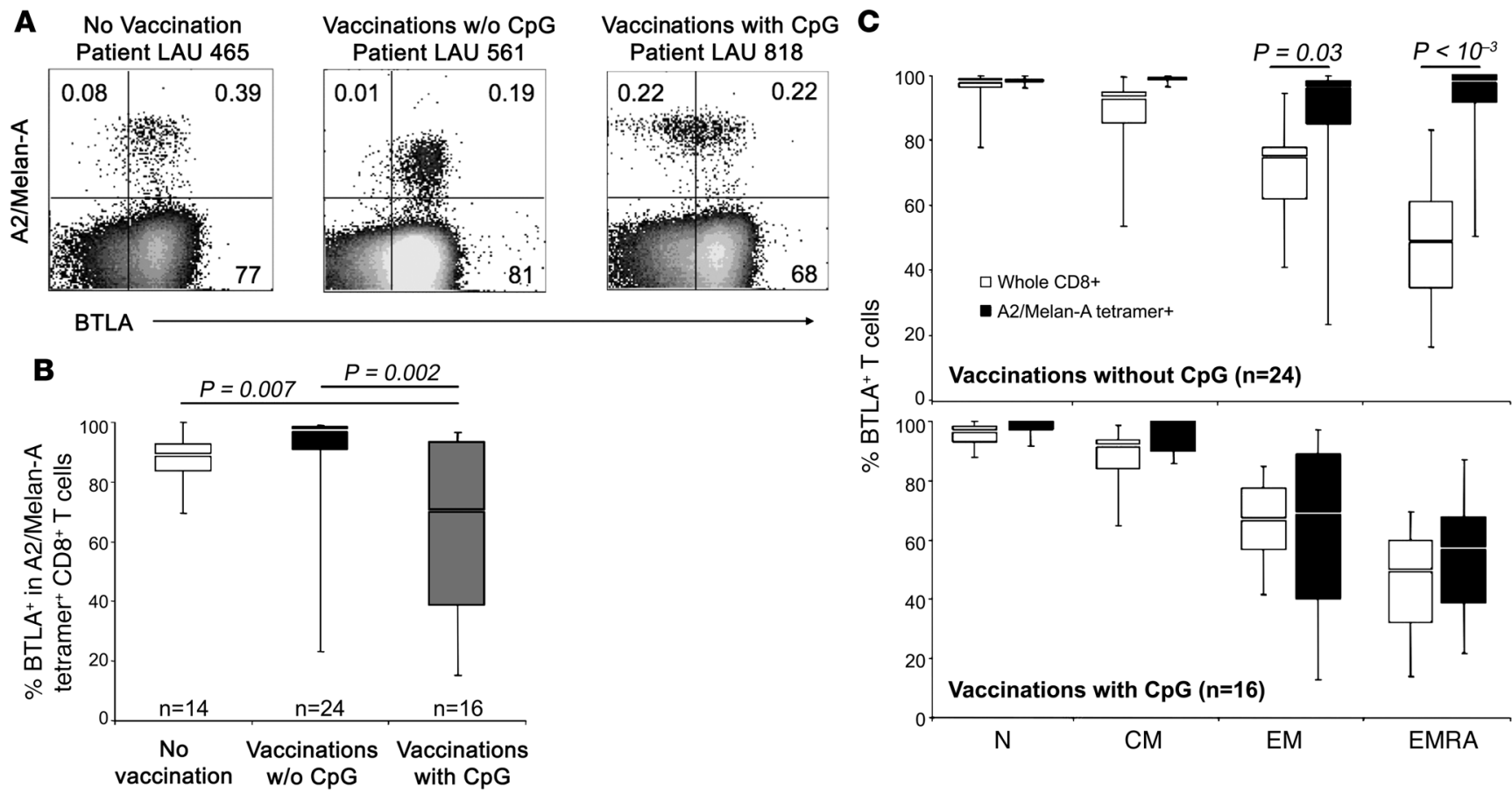

\section{Figure 4}

Repeated vaccination with Melan-AMART-1 peptide, IFA (Montanide ISA-51), and CpG (PF-3512676) induces downregulation of BTLA on Melan-Aspecific CD8 ${ }^{+}$T cells. (A) Representative examples of A2/Melan-AMART-1 tetramer and BTLA staining in unvaccinated and vaccinated patients. Numbers indicate percentages of positive or negative cells. w/o, without. (B) Comparison of BTLA expression in Melan-AMART-1-specific CD8+ T cells from 14 unvaccinated patients, 24 patients vaccinated with peptide but without $\mathrm{CpG}$, and 16 patients vaccinated with peptide plus CpG. (C) Expression of BTLA by Melan-AMART-1-specific CD8 ${ }^{+} \mathrm{T}$ cells (black) and whole $\mathrm{CD}^{+}{ }^{+} \mathrm{T}$ cells (white) according to $\mathrm{CD} 8$ differentiation stages after vaccinations without $\mathrm{CpG}$ (upper panel; $n=24$ ) or with $\mathrm{CpG}$ (lower panel; $n=16$ ). 
A

\section{A Patient vaccinated without CpG}
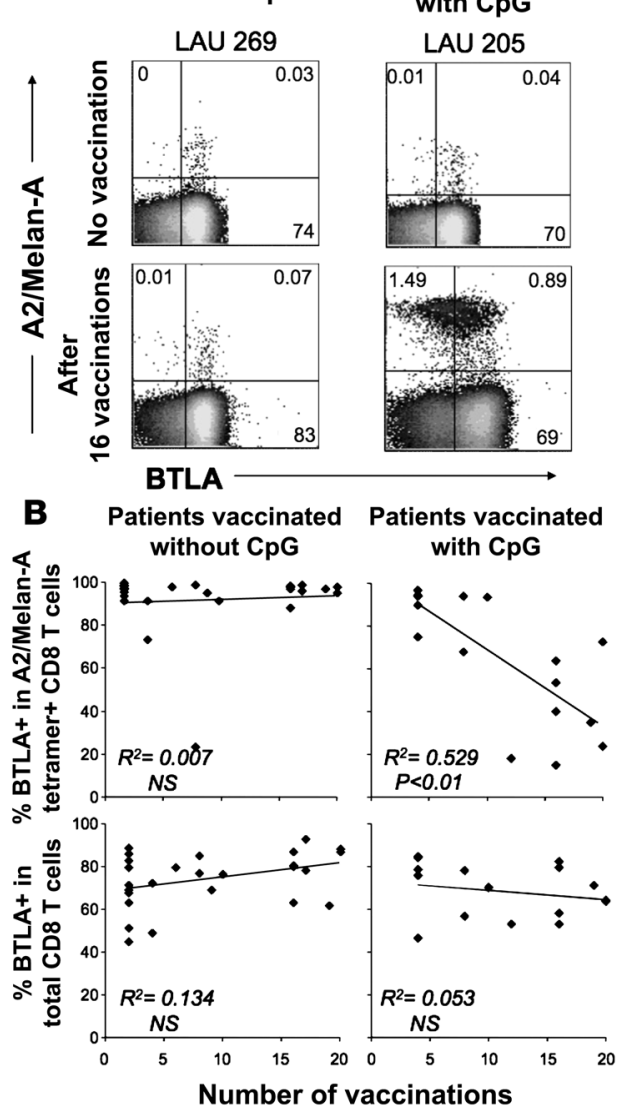

Patient

vaccinated

with CpG

LAU 205
C

C Patients vaccinated Patients vaccinated

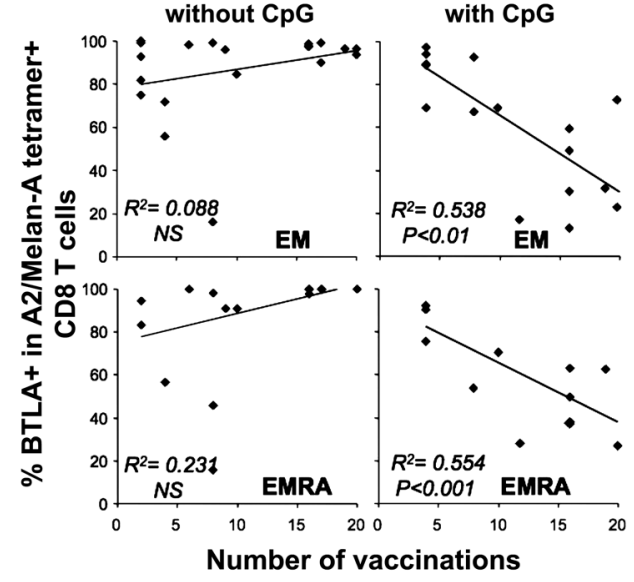

\section{Figure 5}

Progressive BTLA downregulation upon repeated vaccination with CpG. (A) Representative dot plots of A2/Melan-AMART-1 tetramer and BTLA staining (gated on $\mathrm{CD}^{+}{ }^{+} \mathrm{T}$ cells) of PBMCs from 2 patients, before vaccination and after 16 vaccinations, respectively. Numbers indicate percentages of positive or negative cells. (B and $\mathbf{C}$ ) Correlations between numbers of vaccinations without or with $\mathrm{CpG}$ and percentages of $\mathrm{BTLA}^{+} \mathrm{T}$ cells among (B) Melan-A-specific CD8 ${ }^{+} \mathrm{T}$ cells and whole $\mathrm{CD}^{+} \mathrm{T}$ cells and (C) subsets of Melan-Aspecific CD8 ${ }^{+} \mathrm{T}$ cells. cells persistently expressed BTLA, in contrast to influenza-specific $\mathrm{CD}^{+} \mathrm{T}$ cells (Figure 1B). These data suggest that antigenic exposure may result in continued BTLA expression and consequent inhibition of specific $\mathrm{CD}^{+} \mathrm{T}$ cells.

Our data on BTLA and HVEM expression in vivo (Figures 1-3 and Table 1) and the demonstration of T cell inhibition via BTLAHVEM interaction (Figures 3, 6, and 7) suggest that melanomaspecific CD8 responses may be inhibited in vivo via this pathway. Vaccinations with peptide and conventional adjuvants such as IFA did not overcome this problem, as BTLA expression and T cell inhibition remained high (Figure 4).

The introduction of $\mathrm{CpG}$ as an immune adjuvant for human $\mathrm{T}$ cell vaccination shows great promise, indicating that its clinical efficacy should be assessed in clinical phase III trials. CPGs promote expansion of antigen-specific $\mathrm{CD}^{+} \mathrm{T}$ cells to high numbers $(29,32,33)$, with features critical to immune protection such as high $\mathrm{T}$ cell receptor avidity (34), strong clonotypic bursts, and multifunctionality (D.E. Speiser, unpublished observation). We now report that $\mathrm{CPG}$ induces the progressive downregulation of the inhibitory receptor BTLA concurrent with the differentiation to effector cells (Figure 5). This is likely promoted by plasmacytoid DCs that are strongly activated by the triggering of TLR9, the ligand of CPG (35). Since TLR9 is also highly expressed by human $\mathrm{B}$ cells, they may also contribute to the observed profound $\mathrm{T}$ cell activation and differentiation mediated by $\mathrm{CpG}$.

Cancer patients often experience expansion of tumor antigenspecific $\mathrm{CD}^{+} \mathrm{T}$ cells (27), but these remain at relatively low fre- quencies, and their function is significantly decreased in metastases (36). Fortunately, functional T cell deficits in situ are largely reversible (36). T cells appear to readily adapt to changing microenvironments, and their function depends on local activatory and inhibitory signals. Our data demonstrating that BTLA expression and its inhibitory function could be modulated and partly reversed by blocking BTLA triggering support this notion.

Inhibition via BTLA is known to compromise $\mathrm{T}$ cell-mediated immunity in mice $(17,19,21,37,38)$. Our data demonstrate that BTLA inhibits T cell function in humans, as it does in mice, suggesting that BTLA may compromise $\mathrm{T}$ cell-mediated protection from infection and cancer in humans. The functional relevance of BTLA downregulation is at least 2-fold: first, it has direct consequences, abrogating BTLA-mediated inhibition; and second, indirectly, through its correlation with advanced differentiation to highly functional effector $\mathrm{T}$ cells.

Our data show that cancer cells frequently express HVEM in situ. Since tumor-specific T cells may persistently remain highly $\mathrm{BTLA}^{+}$, and still-sizeable proportions of effector $\mathrm{T}$ cells remain $\mathrm{BTLA}^{+}$even in viral infections and after CpG-based vaccination, inhibition via HVEM-BTLA may play an important role in functional $\mathrm{T}$ cell deficits in situ. A previous study showed that HVEM is also frequently expressed by human B cell malignancies and suggested that this finding be further explored for optimization of immunotherapy (39).

BTLA was transiently downregulated upon interaction with HVEM, while stimulation with cognate antigen enhanced BTLA 
A

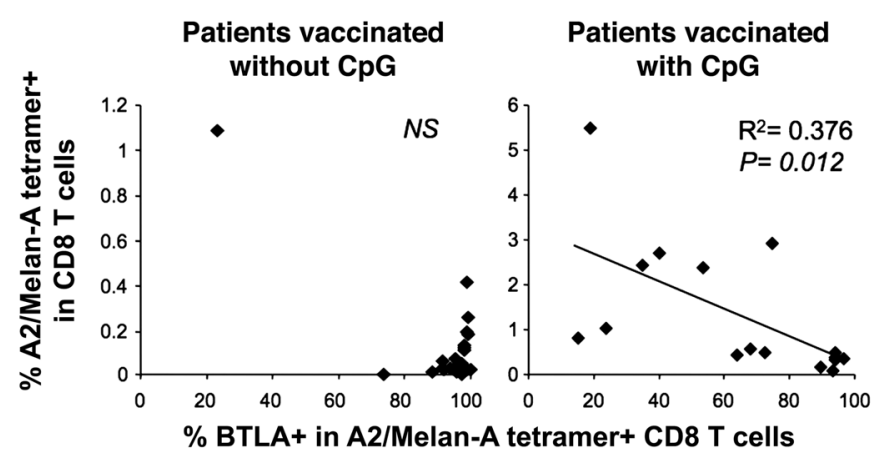

B

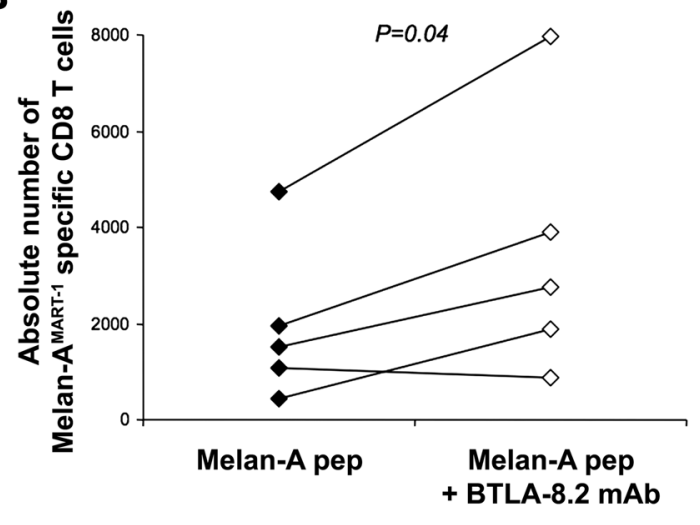

D
C

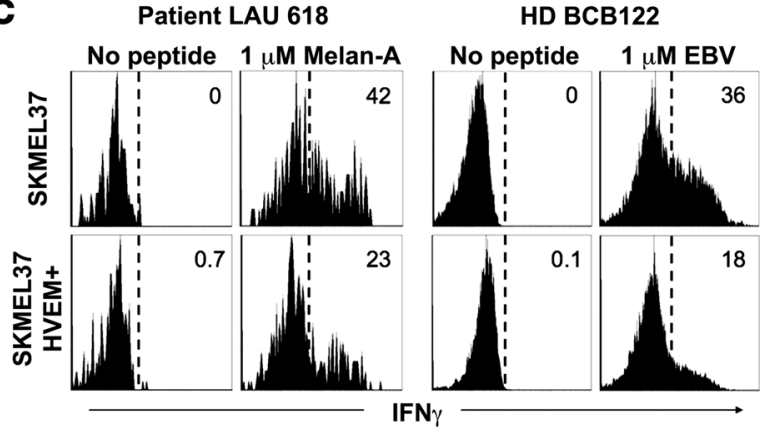

Patient LAU 618

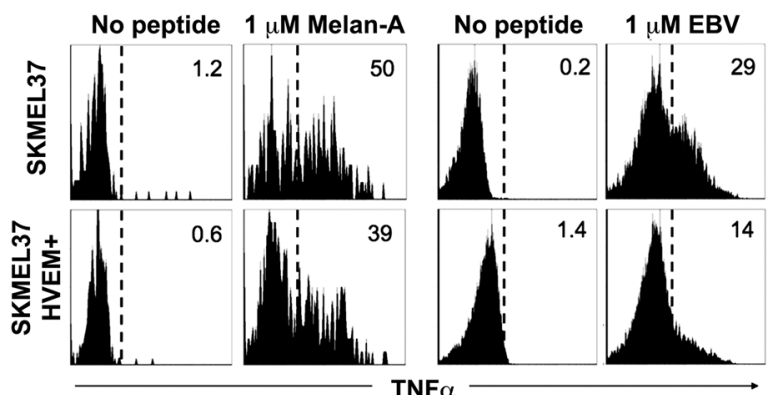

E

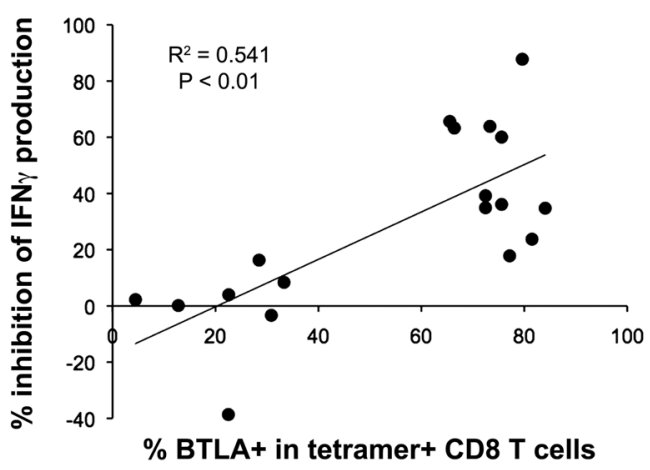

Figure 6

Functional inhibition of $\mathrm{CD}^{+} \mathrm{T}$ cells depending on BTLA expression and HVEM triggering. (A) Direct ex vivo analysis of percentage of A2/Melan-AMART-1 tetramer+ CD8+ ${ }^{+}$cells among PBMCs, in relation to their BTLA expression, after vaccination without and with CpG. (B) In vitro expansion of Melan-AMART-1 tetramer ${ }^{+} T$ cells from healthy donors, after 10 days of stimulation with peptide-loaded DCs, in the absence versus presence of blocking mAb BTLA-8.2. (C and D) IFN- $\gamma$ (C) and TNF- $\alpha$ (D) production by BTLA (C) $^{+}$antigen-specific CD8 ${ }^{+}$T cells after $^{-}$ 4 hours of peptide stimulation of PBMCs assessed ex vivo, i.e., without prior in vitro cultivation. Representative histograms of PBMCs from patient LAU 618 (after 2 vaccinations, with CpG) and from healthy donor BCB122 stimulated by SKMEL37 cells expressing HVEM or not, loaded with Melan-AMART-1 or EBV peptides, respectively. Histograms are gated on tetramer+ T cells. Percentages of cytokine-positive cells are indicated. (E) Significant correlation between percentages of BTLA+ tetramer ${ }^{+} T$ cells and percentages of IFN- $\gamma$ production inhibition (i.e., reduction when stimulated with HVEM+ SKMEL37 cells as compared with HVEM- SKMEL37 cells). Each dot represents a single experiment with Melan-AMART-1_ or EBV-specific T cells.

expression in the absence of HVEM (Figure 7, C and D). A similar effect has been shown for HVEM, which is downregulated after binding to LIGHT (40). It remains to be determined whether the reduced BTLA expression that we observed after vaccination with $\mathrm{CPG}$ is due to enhanced binding to HVEM in vivo. Alternatively, BTLA expression may be regulated in conjunction with $\mathrm{CD}^{+} \mathrm{T}$ cell differentiation, in parallel with the progressively reduced expression of CD27 and CD28 (Supplemental Figure 4A) by advanced differentiated effector $\mathrm{T}$ cells.
HVEM interacts not only with BTLA but also with CD160, LIGHT, and lymphotoxin- $\alpha$ (41-43). While ligation of HVEM to LIGHT and lymphotoxin- $\alpha$ stimulates $\mathrm{T}$ cell responses (44), HVEM binding to BTLA and CD160 delivers co-inhibitory signals to T cells $(11,12,42,45)$. Thus, HVEM is a bidirectional molecular switch regulating $\mathrm{T}$ cell activation by co-stimulation or co-inhibition whose outcome depends on the ligand engaged (41). However, the overall function of HVEM is inhibitory (46), suggesting that negative signals mediated by BTLA and CD160 are dominant. 

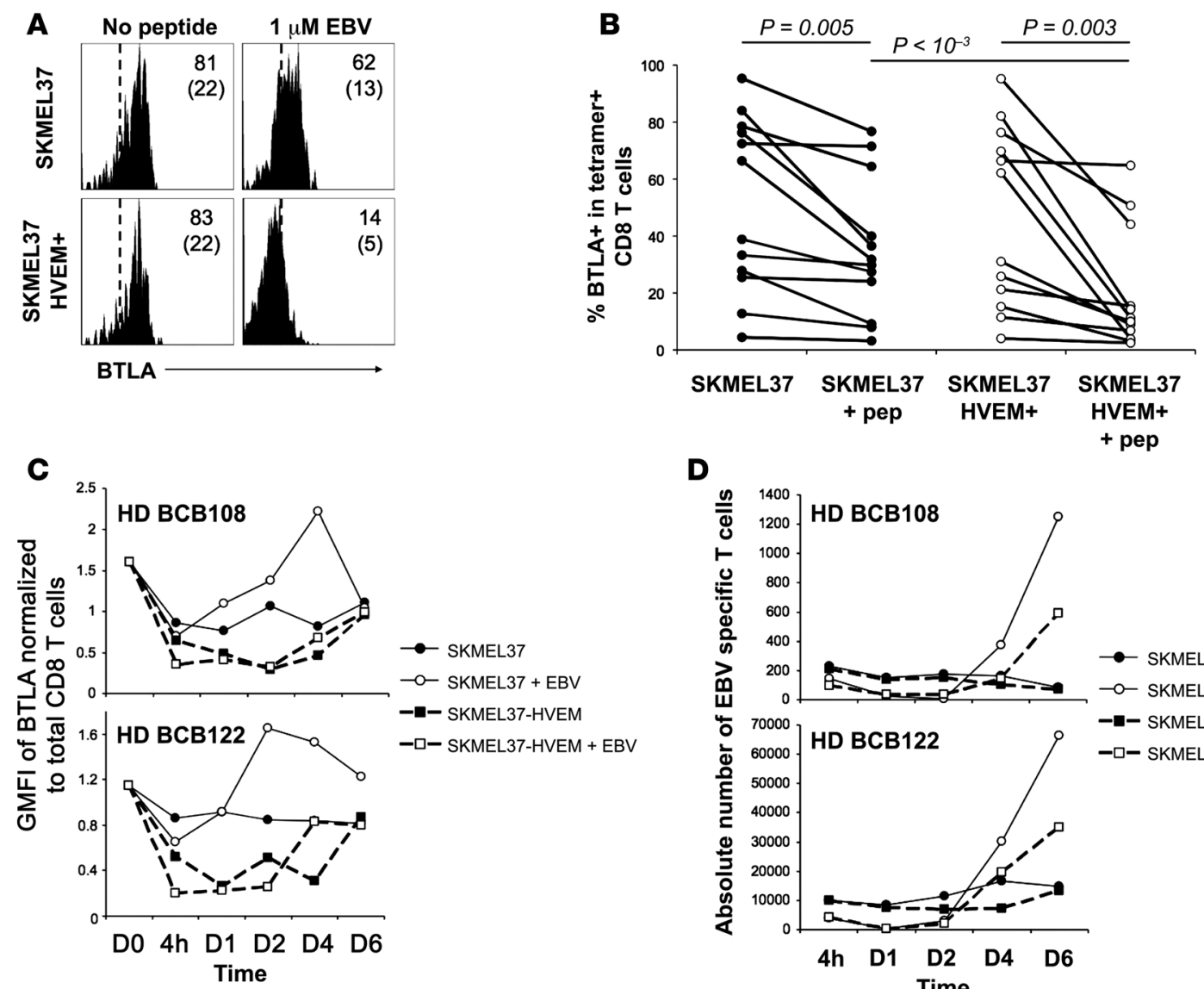

D

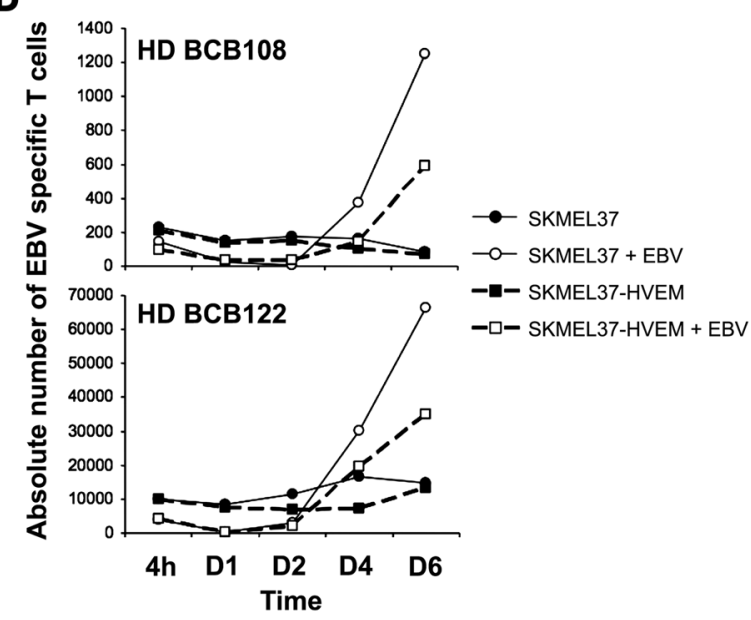

Figure 7

Downregulation of BTLA by primary CD8 ${ }^{+} \mathrm{T}$ cells upon $\mathrm{T}$ cell receptor activation and HVEM ligation. (A) Representative example of BTLA downregulation by $B T L A^{+} E B V$-specific $C D 8^{+} T$ cells from PBMCs of healthy donor BCB136 upon EBV peptide stimulation with target cells expressing HVEM or not. Histograms are gated on $\mathrm{CD}^{+} \mathrm{EBV}^{+} \mathrm{T}$ cells. Percentages of BTLA ${ }^{+}$cells (and GMFI in parentheses) are indicated. (B) Percentages of BTLA expression by antigen-specific CD8+ $T$ cells after stimulation with SKMEL37 cells expressing HVEM or not. Each dot represents a single experiment with Melan-AMART-1 - or EBV-specific T cells. (C and D) Kinetic analysis of surface BTLA expression (C) and expansion (D) of EBV-specific CD8+ T cells upon stimulation with irradiated target cells expressing HVEM or not, in the presence or absence of EBV peptide. Results for 2 of 5 representative healthy donors tested are shown.

Other cell functions may also be affected, since BTLA or CD160 triggering may also promote cell survival (47). The complex interactions within this receptor system require further investigation. Nevertheless, it seems clear that negative signals delivered by BTLA may be relevant even without interfering with CD160. Possibly, simultaneous blockade of both, BTLA and CD160, may result in an even greater promotion of $\mathrm{T}$ cell activation (41).

Therapeutic blockade of the inhibitory pathway mediated by PD-1/PD-L1 has been shown to augment responses of otherwise exhausted T cells (4), an effect that was further enhanced upon double blockade of PD-1 and LAG-3 (7). Blockade of BTLA provides an additional therapeutic option, possibly already prominent during the critical early phases of immune responses, since BTLA is strongly expressed in naive T cells, in contrast to PD-1 and LAG-3 $(4,11,24,25,48)$. A role of BTLA in naive T cells is supported by our observation that blocking BTLA-HVEM ligation during priming of naive $\mathrm{CD}^{+} \mathrm{T}$ cells may have contributed to the enhanced proliferation (Figure 6B).

In summary, our findings provide a basis for improving current therapies aimed at avoiding BTLA-mediated $\mathrm{T}$ cell inhibition in infectious and malignant diseases and promoting $\mathrm{T}$ cell inhibition in autoimmune diseases or after transplantation.

\section{Methods}

Patients and vaccination. HLA-A*0201-positive patients with stage III/IV metastatic melanoma were included in the Ludwig Institute for Cancer Research clinical trials LUD 96-010 and LUD 00-018, approved by institutional review boards and regulatory agencies $(28,29)$. Patients received monthly low-dose vaccinations s.c. with $100 \mu \mathrm{g}$ Melan-A MART-1 peptide. CpG (500 ug PF-3512676/7909; provided by Pfizer/Coley Pharmaceutical Group) was added where indicated. For all patients, vaccines were formulated with IFA (300-600 $\mu$ l Montanide ISA-51), except for 6 of 13 patients in the group "without CpG" who received no adjuvant (2 patients) or QS21 plus MPL (4 patients) $(28,29)$.

HLA-A2/peptide tetramers and flow cytometry analysis of primary (not cultured) T cells. PBMCs were obtained by density centrifugation using Lymphoprep (Axis-Shieldy) and cryopreserved in RPMI 1640 supplemented with $40 \%$ FCS and $10 \%$ DMSO. HLA-A $* 201$ /peptide tetramers were prepared by P. Guillaume and I. Luescher as described (27) with modified Melan- $\mathrm{A}_{26-35} \mathrm{~A} 27 \mathrm{~L}$ peptide ELAGIGILTV, EBV lytic protein BMLF1 
peptide GLCTLVAML, CMV late antigen pp65 peptide NLVPMVATV, or influenza virus matrix protein peptide GILGFVFTL. Five-color stains were done with APC- and PE-conjugated A2/peptide tetramers, ECD-conjugated anti-CD45RA (Beckman Coulter), PE-conjugated anti-BTLA, PE-Cy7conjugated anti-CCR7, and Pacific Blue-conjugated anti-CD8 antibodies (BD). Analysis of primary T cells was performed as follows: PBMCs were thawed, then positively enriched with anti-CD8-coated magnetic microbeads (Miltenyi Biotec), which resulted in a population consisting of more than $90 \% \mathrm{CD}^{+} \mathrm{T}$ lymphocytes. Cells were stained first with tetramers for 30 minutes at $4^{\circ} \mathrm{C}$ in PBS, $0.2 \%$ BSA, and $50 \mathrm{mM}$ EDTA and then with antibodies as indicated ( 30 minutes, $4^{\circ} \mathrm{C}$ ). Cells were acquired on a FACS LSRII and analyzed with Diva software (BD). Using a new antibody specific for BTLA labeled with Alexa Fluor 647 (BTLA-7.1 mAb; Supplemental Figure 2B), we confirmed the results obtained with the BTLA-specific antibody from BD (Supplemental Figure 7 and data not shown).

Preparation of mature DCs and stimulation of Melan- $A^{\text {MART-1 }}$-specific $C D 8^{+} T$ cells. CD $14^{+}$monocytes were immunomagnetically purified with CD14 mAb-conjugated microbeads (Miltenyi Biotec) from PBMCs of healthy donors. $\mathrm{CD} 14^{+}$monocytes were cultured for 2 days in 6 -well plates at $2 \times 10^{6}$ cells/well in RPMI 1640 medium with 8\% human serum, 1,000 U/ml GM-CSF (Immunotools), and $20 \mathrm{ng} / \mathrm{ml} \mathrm{IL-4} \mathrm{(R \& D} \mathrm{Systems).} \mathrm{LPS}(5 \mu \mathrm{g} / \mathrm{ml})$ was added for 1 additional day of culture. After 3 days, mature DCs were harvested and loaded with Melan-AMART-1 peptide (ELAGIGILTV) during 1 hour at $37^{\circ} \mathrm{C}$. After washing, mature DCs were cocultured with immunomagnetically purified autologous $\mathrm{CD} 8^{+} \mathrm{T}$ cells, with or without blocking $\mathrm{mAb}$ BTLA-8.2 $(10 \mu \mathrm{g} / \mathrm{ml})$. Flow cytometry with A2/Melan-A ${ }^{\text {MART-1 }}$ tetramers was performed after 10 days of culture.

Cytokine production by primary T cells, assessed by flow cytometry. SKMEL37 melanoma cell line (HLA-A*0201-positive, Melan-AM ${ }^{\text {ART-1 }}$-negative) was transfected with a full-length human HVEM (gift from P. Schneider, University of Lausanne, Lausanne, Switzerland) inserted into PCR3 expression vector or with empty PCR3, using FuGENE HD (Roche) transfection reagent according to the manufacturer's instructions. Cells were cultured in the presence of $0.5 \mathrm{mg} / \mathrm{ml} \mathrm{G} 418 \mathrm{~s}$ (Sigma-Aldrich). PBMCs were thawed the day before the experiment and cultured overnight in RPMI containing $8 \%$ human serum. Magnetic microbead-sorted $\mathrm{CD}^{+} \mathrm{T}$ cells were stained with A2/EBV or A2/Melan-AMART-1 tetramers. SKMEL37 cells transfected with HVEM or with empty vector were loaded 1 hour at $37^{\circ} \mathrm{C}$ with $1 \mu \mathrm{M}$ Melan-AMART-1 (ELAGIGILTV) or EBV (GLCTLVAML) peptides and washed. Then, $0.1 \times 10^{6}$ $\mathrm{CD}^{+} \mathrm{T}$ cells were cocultured with $5 \times 10^{4}$ irradiated SKMEL37 cells in the presence of $10 \mu \mathrm{g} / \mathrm{ml}$ Brefeldin A (Sigma-Aldrich) and PE-CY5-conjugated anti-CD107a (BD). After different time points, $\mathrm{CD}^{+}$cells were stained with tetramers and then with Pacific Blue-conjugated anti-CD8 and PE-conjugated anti-BTLA (BD). After washing in PBS, cells were stained with LIVE/DEAD Fixable NEAR-IR (Invitrogen), in order to exclude dead cells, and fixed at room temperature (RT) during 30 minutes with $1 \%$ paraformaldehyde buffer. Cells were washed and stained with mAbs antiIFN- $\gamma$-PE-CY7 and anti-TNF- $\alpha$-FITC (BD) in FACS buffer with $0.1 \%$ saponin for 30 minutes at RT. Cells were then analyzed on a FACS LSRII.

Characterization of new BTLA-specific antibodies (BTLA-7.1 and-8.2) and blocking of BTLA-HVEM interaction. Cos cells $\left(2 \times 10^{5}\right)$ transiently transfected for 24 hours with a construct encoding for human BTLA were treated with a mixture of $10 \mu \mathrm{g} / \mathrm{ml}$ of HVEM-FC and a range of concentrations $(0.01-30 \mu \mathrm{g} / \mathrm{ml})$ of BTLA-7.1 or $-8.2 \mathrm{mAb}$ for 30 minutes at $4^{\circ} \mathrm{C}$. Cells were then washed with PBS and stained with R-PE-conjugated AffiniPure
$\mathrm{F}\left(\mathrm{ab}^{\prime}\right)_{2}$ Fragment Goat Anti-Human IgG $(\mathrm{H}+\mathrm{L})$ (Jackson ImmunoResearch Laboratories Inc.) for 15 minutes at $4{ }^{\circ} \mathrm{C}$. Binding of HVEM-Fc and blocking activity of BTLA-7.1 or $-8.2 \mathrm{mAb}$ were determined by flow cytometry on a BD FACScan cytometer. To assess BTLA-HVEM interaction, $5 \times 10^{3}$ Me 275 or Me 290 melanoma cells were cocultured with $10 \times 10^{3} \mathrm{~T}$ cell clones specific for Melan-A, in the presence of $10 \mu \mathrm{g} / \mathrm{ml}$ blocking anti-BTLA antibody or IgG1 isotype control antibody (Beckman Coulter). After 24 hours of incubation, IFN- $\gamma$ concentrations in supernatants were then measured by ELISA.

Immunohistochemistry. Consecutive paraffin sections ( $4 \mu \mathrm{M}$ thick) were cut and mounted on electrostatically precharged slides (Superfrost Plus, Menzel-Gläser). The sections were deparaffinized in xylene and rehydrated through graded alcohols. After endogenous peroxidase quenching $\left(0.3 \% \mathrm{H}_{2} \mathrm{O}_{2}\right.$ in distilled water for 5 minutes), antigens were retrieved by boiling the sections in $1 \mathrm{mM}$ EDTA solution, $\mathrm{pH} 9.0$, in a pressure cooker for 3 minutes. Tissue sections were incubated overnight at $4{ }^{\circ} \mathrm{C}$ with rat anti-human HVEM antibody (provided by O. Donze, Alexis Biochemicals). After washing, tissue sections were incubated for 30 minutes at RT with horseradish peroxidase-conjugated anti-rat IgG (Dako) and diaminobenzidine (Dako). Finally, the sections were counterstained with hematoxylin and dehydrated through graded alcohols and xylene.

Statistics. Figures showing BTLA expression are box-and-whisker plot representations. Boxes indicate 25 th and 75 th percentiles. The whiskers represent maximum and minimum values, whereas the horizontal bars within the box represent median values. For comparisons of data from healthy donors and patients (e.g., Figure 2B), the 2-sample $t$ test was used assuming independent samples and unknown unequal variances for the underlying populations. When comparing different populations between patients or between healthy individuals, a 2 -tailed paired $t$ test was used (e.g., Figure 1B). The Pearson correlation test was used to demonstrate significant inverse correlations between BTLA expression and numbers of vaccinations (Figure 5). $P$ values less than 0.05 were considered significant.

\section{Acknowledgments}

We are obliged to the patients and blood donors for their dedicated collaboration. We gratefully acknowledge A. Krieg, P. Baumgaertner, H. Bouzourene, M.B. Irving, N. Rufer, P. Schneider, and V. Voelter for collaboration and advice. We thank I. Luescher, P. Guillaume, and O. Donze for reagents and Pfizer and Coley Pharmaceutical Group for pharmaceutical products. We are also thankful for the excellent help of C. Geldhof, L. Leyvraz, N. Montandon, and M. van Overloop. This study was supported by G. Michlin, the Ludwig Institute for Cancer Research, the Swiss Cancer League/Oncosuisse, the Swiss National Science Foundation, the Swiss National Center of Competence in Research (NCCR) Molecular Oncology, the Cancer Research Institute (United States), the Atlantic Philanthropies (United States), and the Wilhelm Sander Foundation (Germany).

Received for publication June 3, 2009, and accepted in revised form October 7, 2009.

Address correspondence to: Daniel E. Speiser, Ludwig Institute for Cancer Research, Hôpital Orthopédique, Niveau 5 Est, Av. PierreDecker 4, CH-1011 Lausanne, Switzerland. Phone: 41-21-314-0182; Fax: 41-21-314-74-77; E-mail: daniel.speiser@hospvd.ch.

\footnotetext{
1. Bretscher PA. A two-step, two-signal model for the primary activation of precursor helper T cells. Proc Natl Acad Sci U S A. 1999;96(1):185-190.

2. Chambers CA, Allison JP. Costimulatory regulation of $\mathrm{T}$ cell function. Curr Opin Cell Biol.
}

1999;11(2):203-210.

3. Chambers CA, Kuhns MS, Egen JG, Allison JP. CTLA-4-mediated inhibition in regulation of $\mathrm{T}$ cell responses: mechanisms and manipulation in tumor immunotherapy. Annu Rev Immunol.
2001;19:565-594.

4. Barber DL, et al. Restoring function in exhausted CD8 T cells during chronic viral infection. Nature. 2006;439(7077):682-687.

5. Ha SJ, West EE, Araki K, Smith KA, Ahmed R. 
Manipulating both the inhibitory and stimulatory immune system towards the success of therapeutic vaccination against chronic viral infections. Immunol Rev. 2008;223:317-333.

6. Peggs KS, Quezada SA, Allison JP. Cell intrinsic mechanisms of T-cell inhibition and application to cancer therapy. Immunol Rev. 2008;224:141-165.

7. Blackburn SD, et al. Coregulation of CD8+ T cell exhaustion by multiple inhibitory receptors during chronic viral infection. Nat Immunol. 2009;10(1):29-37.

8. McMahon CW, Raulet DH. Expression and function of NK cell receptors in CD8+ T cells. Curr Opin Immunol. 2001;13(4):465-470.

9. Speiser DE, et al. In vivo expression of natural killer cell inhibitory receptors by human melanoma-specific cytolytic T lymphocytes. J Exp Med. 1999;190(6):775-782.

10. Murphy K, Nelson C, Šedý J. Balancing co-stimulation and inhibition with BTLA and HVEM. Nat Rev Immunol. 2006;6(9):671-681.

11. Sedy JR, et al. B and T lymphocyte attenuator regulates $\mathrm{T}$ cell activation through interaction with herpesvirus entry mediator. Nat Immunol. 2005;6(1):90-98.

12. Watanabe $\mathrm{N}$, et al. BTLA is a lymphocyte inhibitory receptor with similarities to CTLA-4 and PD-1. Nat Immunol. 2003;4(7):670-679.

13. Chemnitz JM, Lanfranco AR, Braunstein I, Riley JL. B and T lymphocyte attenuator-mediated signal transduction provides a potent inhibitory signal to primary human CD4 T cells that can be initiated by multiple phosphotyrosine motifs. J Immunol. 2006;176(11):6603-6614.

14. Gavrieli M, Murphy KM. Association of Grb-2 and PI3K p85 with phosphotyrosile peptides derived from BTLA. Biochem Biophys Res Commun. 2006;345(4):1440-1445.

15. Vendel AC, Calemine-Fenaux J, Izrael-Tomasevic A, Chauhan V, Arnott D, Eaton DL. B and T lymphocyte attenuator regulates B cell receptor signaling by targeting Syk and BLNK. J Immunol. 2009;182(3):1509-1517.

16. Krieg C, Boyman O, Fu Y, Kaye J. B and T lymphocyte attenuator regulates CD8 $+\mathrm{T}$ cell-intrinsic homeostasis and memory cell generation. Nat Immunol. 2007;8(2):162-171.

17. Lepenies B, et al. Ligation of B and T lymphocyte attenuator prevents the genesis of experimental cerebral malaria. J Immunol. 2007;179(6):4093-4100.

18. Deppong C, et al. Cutting edge: B and T lymphocyte attenuator and programmed death receptor-1 inhibitory receptors are required for termination of acute allergic airway inflammation. J Immunol. 2006;176(7):3909-3913.

19. Oya Y, et al. Development of autoimmune hepati- tis-like disease and production of autoantibodies to nuclear antigens in mice lacking B and T lymphocyte attenuator. Arthritis Rheum. 2008;58(8):2498-2510.

20. Liu X, et al. Cutting edge: a critical role of B and T lymphocyte attenuator in peripheral $\mathrm{T}$ cell tolerance induction. J Immunol. 2009;182(8):4516-4520.

21. Sun Y, et al. B and T lymphocyte attenuator tempers early infection immunity. J Immunol. 2009;183(3):1946-1951.

22. Okano M, et al. Allergen-specific immunotherapy alters the expression of $\mathrm{B}$ and $\mathrm{T}$ lymphocyte attenuator, a co-inhibitory molecule, in allergic rhinitis. Clin Exp Allergy. 2008;38(12):1891-1900.

23. Pittet MJ, et al. High frequencies of naive MelanA/MART-1-specific CD8(+) T cells in a large proportion of human histocompatibility leukocyte antigen (HLA)-A2 individuals. J Exp Med. 1999;190(5):705-715.

24. Han P, Goularte OD, Rufner K, Wilkinson B, Kaye J. An inhibitory Ig superfamily protein expressed by lymphocytes and APCs is also an early marker of thymocyte positive selection. J Immunol. 2004;172(10):5931-5939.

25. Otsuki N, Kamimura Y, Hashiguchi M, Azuma M. Expression and function of the $\mathrm{B}$ and $\mathrm{T}$ lymphocyte attenuator (BTLA/CD272) on human T cells. Biochem Biophys Res Commun. 2006;344(4):1121-1127.

26. Sallusto F, Lenig D, Forster R, Lipp M, Lanzavecchia A. Two subsets of memory $T$ lymphocytes with distinct homing potentials and effector functions. Nature. 1999;401(6754):708-712.

27. Romero P, et al. Ex vivo staining of metastatic lymph nodes by class I major histocompatibility complex tetramers reveals high numbers of antigen-experienced tumor-specific cytolytic T lymphocytes. J Exp Med. 1998;188(9):1641-1650.

28. Lienard D, et al. Ex vivo detectable activation of Melan-A-specific T cells correlating with inflammatory skin reactions in melanoma patients vaccinated with peptides in IFA. Cancer Immun. 2004;4:4.

29. Speiser DE, et al. Rapid and strong human CD8+ $\mathrm{T}$ cell responses to vaccination with peptide, IFA, and $\mathrm{CPG}$ oligodeoxynucleotide 7909. J Clin Invest. 2005;115(3):739-746.

30. Betts MR, et al. Sensitive and viable identification of antigen-specific CD8+ T cells by a flow cytometric assay for degranulation. J Immunol Methods. 2003;281(1-2):65-78.

31. Blankenstein $\mathrm{T}$. The role of tumor stroma in the interaction between tumor and immune system. Curr Opin Immunol. 2005;17(2):180-186.

32. Kochenderfer JN, Chien CD, Simpson JL, Gress RE. Maximizing CD8+ T cell responses elicited by peptide vaccines containing $\mathrm{CpG}$ oligodeoxynucleotides. Clin Immunol. 2007;124(2):119-130.
33. Miconnet I, et al. CpG are efficient adjuvants for specific CTL induction against tumor antigen-derived peptide. J Immunol. 2002;168(3):1212-1218.

34. Speiser DE, et al. Unmodified self antigen triggers human CD8 T cells with stronger tumor reactivity than altered antigen. Proc Natl Acad Sci U S A. 2008;105(10):3849-3854.

35. Krieg AM. Development of TLR9 agonists for cancer therapy. J Clin Invest. 2007;117(5):1184-1194.

36. Zippelius A, et al. Effector function of human tumor-specific CD8 T cells in melanoma lesions: a state of local functional tolerance. Cancer Res. 2004;64(8):2865-2873.

37. Pierer $M$, et al. Herpesvirus entry mediator-Ig treatment during immunization aggravates rheumatoid arthritis in the collagen-induced arthritis model. J Immunol. 2009;182(5):3139-3145.

38. Steinberg MW, et al. A crucial role for HVEM and BTLA in preventing intestinal inflammation. J Exp Med. 2008;205(6):1463-1476.

39. Costello RT, et al. Stimulation of non-Hodgkin's lymphoma via HVEM: an alternate and safe way to increase Fas-induced apoptosis and improve tumor immunogenicity. Lenkemia. 2003;17(12):2500-2507.

40. Morel Y, et al. Reciprocal expression of the TNF family receptor herpes virus entry mediator and its ligand LIGHT on activated T cells: LIGHT down-regulates its own receptor. J Immunol. 2000;165(8):4397-4404.

41. Cai G, Freeman GJ. The CD160, BTLA, LIGHT/ HVEM pathway: a bidirectional switch regulating Tcell activation. Immunol Rev. 2009;229(1):244-258.

42. Cai G, et al. CD160 inhibits activation of human $\mathrm{CD} 4+\mathrm{T}$ cells through interaction with herpesvirus entry mediator. Nat Immunol. 2008;9(2):176-185.

43. Zhai Y, et al. LIGHT, a novel ligand for lymphotoxin beta receptor and TR2/HVEM induces apoptosis and suppresses in vivo tumor formation via gene transfer. J Clin Invest. 1998;102(6):1142-1151.

44. Ware CF. Targeting lymphocyte activation through the lymphotoxin and LIGHT pathways. Immunol Rev. 2008;223:186-201.

45. Krieg C, Han P, Stone R, Goularte OD, Kaye J. Functional analysis of $\mathrm{B}$ and $\mathrm{T}$ lymphocyte attenuator engagement on CD4+ and CD8+ T cells. J Immunol. 2005;175(10):6420-6427.

46. Wang Y, et al. The role of herpesvirus entry mediator as a negative regulator of $\mathrm{T}$ cell-mediated responses. J Clin Invest. 2005;115(3):711-717.

47. Cheung TC, et al. Unconventional ligand activation of herpesvirus entry mediator signals cell survival. Proc Natl Acad Sci US A. 2009;106(15):6244-6249.

48. Hurchla MA, et al. B and T lymphocyte attenuator exhibits structural and expression polymorphisms and is highly Induced in anergic CD4+ T cells. J Immunol. 2005;174(6):3377-3385. 